\title{
Diabetes mellitus and chronic kidney disease in the Eastern Mediterranean Region: findings from the Global Burden of Disease 2015 study
}

\author{
GBD 2015 Eastern Mediterranean Region Diabetes and Chronic Kidney Disease \\ Collaborators $^{1}$
}

Received: 1 May 2017/Revised: 30 June 2017/ Accepted: 1 July 2017/Published online: 3 August 2017

(c) The Author(s) 2017. This article is an open access publication

\begin{abstract}
Objectives We used findings from the Global Burden of Disease 2015 study to update our previous publication on the burden of diabetes and chronic kidney disease due to diabetes (CKD-DM) during 1990-2015.

Methods We extracted GBD 2015 estimates for prevalence, mortality, and disability-adjusted life years (DALYs) of diabetes (including burden of low vision due to diabetes, neuropathy, and amputations and CKD-DM for 22 countries of the EMR from the GBD visualization tools. Results In 2015, 135,230 (95\% UI 123,034-148,184) individuals died from diabetes and 16,470 (95\% UI 13,977-18,961) from CKD-DM, 216 and 179\% increases, respectively, compared to 1990 . The total number of people with diabetes was 42.3 million (95\% UI 38.6-46.4 million) in 2015. DALY rates of diabetes in 2015 were significantly higher than the expected rates based on Sociodemographic Index (SDI).
\end{abstract}

This article is part of the supplement "The state of health in the Eastern Mediterranean Region, 1990-2015".

The members of GBD (Global Burden of Disease) 2015 Eastern Mediterranean Region Diabetes and Chronic Kidney Disease Collaborators are listed at the end of the article. Ali H. Mokdad, on behalf of GBD 2015 Eastern Mediterranean Region Diabetes and Chronic Kidney Disease Collaborators, is the corresponding author.

Electronic supplementary material The online version of this article (doi:10.1007/s00038-017-1014-1) contains supplementary material, which is available to authorized users.

GBD 2015 Eastern Mediterranean Region Diabetes and Chronic Kidney Disease Collaborators

mokdaa@uw.edu

1 Institute for Health Metrics and Evaluation, University of Washington, Seattle, WA, USA
Conclusions Our study showed a large and increasing burden of diabetes in the region. There is an urgency in dealing with diabetes and its consequences, and these efforts should be at the forefront of health prevention and promotion.

Keywords Diabetes - Chronic kidney disease - Burden of disease $\cdot$ Eastern Mediterranean Region

\section{Introduction}

Diabetes is an important cause of disability and death around the world and is a major risk factor for other diseases (GBD 2015 DALYs/HALE Collaborators 2016; Moradi-Lakeh et al. 2016b). The World Health Organization Eastern Mediterranean Region (EMR) has the highest age-standardized rate of disability-adjusted life years (DALYs) from diabetes (GBD 2015 DALYs/HALE Collaborators 2016; Institute for Health Metrics and Evaluation 2016). Analysis of the global burden of disease (GBD) 2013 study showed that the increasing burden of diabetes in the EMR in recent decades is beyond that expected based on the demographic changes of population growth and aging, and is also due to increases in age-specific DALY rates (Mokdad et al. 2016; Moradi-Lakeh et al. 2016b). This increasing trend has been reported by other studies as well (Sozmen et al. 2015) and is mainly because of the epidemics of obesity and physical inactivity as the main risk factors for type 2 diabetes mellitus (Mokdad et al. 2014, 2016; Sozmen et al. 2015).

Tracking of personal health spending in the United States shows that diabetes imposes the highest health care spending (Dieleman et al. 2016). International Diabetes Federation estimated US \$17.1-27.7 billion is spent 
annually in the Middle East and North Africa on diabetes, an amount which is expected to double by 2040 (IDF 2015). In this report, we present estimates of the burden of diabetes mellitus and chronic kidney disease due to diabetes mellitus (CKD-DM) from the Global Burden of Disease 2015 study.

\section{Methods}

GBD 2015 covers 195 countries, 21 regions, and seven super-regions from 1990 to 2015 for 315 diseases and injuries, 2619 sequelae, and 79 risk factors by age and sex. Detailed descriptions of GBD 2015 methodology and specific diabetes mellitus methodology have been provided elsewhere (GBD 2015 DALYs/HALE Collaborators 2016; GBD 2015 Disease and Injury Incidence and Prevalence Collaborators 2016; Duncan et al. 2017; Moradi-Lakeh et al. 2016b; GBD 2015 Causes of Death Collaborators 2016).

We evaluated the burden of diabetes and CKD-DM in 22 EMR countries: Afghanistan, Bahrain, Djibouti, Egypt, Iran, Iraq, Jordan, Kuwait, Lebanon, Libya, Morocco, Pakistan, Palestine, Qatar, Saudi Arabia, Somalia, Sudan, Syria, Tunisia, United Arab Emirates (UAE) and Yemen. The total population of the EMR is over 580 million people.

Diabetes mellitus in GBD is considered both as a disease and a metabolic risk factor. In this study, we focus on its burden as a disease. The burden of uncomplicated diabetes, vision loss caused by diabetes (moderate low vision, severe low vision, and blindness), diabetic neuropathy, diabetic foot due to neuropathy, and amputation are included in the burden of diabetes (Duncan et al. 2017; Moradi-Lakeh et al. 2016b). Also, we estimated burden of CKD-DM as part of the chronic kidney disease burden.

All-cause mortality envelopes (total number of deaths) were first estimated for each country during the period of 1990-2015. For this purpose, we used all accessible data from vital registration systems, sibling history surveys, sample registration data, and household recall of deaths. We extracted causes of death data from the same sources, as well as available verbal autopsies, and then used cause of death ensemble modeling to estimate the number of deaths from diabetes and CKD-DM by age, sex, country, and year (GBD 2015 DALYs/HALE Collaborators 2016; Duncan et al. 2017; Moradi-Lakeh et al. 2016b). In this approach, a large variety of possible models are explored to estimate trends in causes of death. Possible models are identified based on a covariate selection algorithm that yields several plausible combinations of covariates; they are then run through different model classes, including mixed effects linear models and spatiotemporal Gaussian process regression models for cause fractions and death rates. All models for each cause of death are then assessed using out-of-sample predictive validity and combined into an ensemble with optimal out-of-sample predictive performance (Foreman et al. 2012).

We updated our previous systematic review for the GBD study separately for non-fatal outcomes of diabetes mellitus and CKD-DM. Data on incidence, prevalence, and excess mortality were extracted from data sources. We assumed no remission for diabetes. Bayesian meta-regression analysis through DisMod-MR 2.1 was used for disease modeling. Model-based epidemiological estimates in combination with disability weights were used to calculate cause-specific years lived with disability (YLDs) for each age, sex, location, and year. DALYs were calculated through summation of years of life lost (YLLs) and YLDs (GBD 2015 DALYs/HALE Collaborators 2016; GBD 2015 Disease and Injury Incidence and Prevalence Collaborators 2016).

In GBD 2015, we used country-location estimates of a composite Socio-demographic Index (SDI) based on the geometric mean of income per capita, average years of schooling in individuals older than 15 years, and total fertility rate. The numbers were rescaled to a number between zero and one, based on highest and lowest country-location measures. In 2015, SDI had a range between 0.1506 (Somalia) and 0.8747 (United Arab Emirates) in the EMR. We used SDI to estimate expected burden for each disease based on the demographic and social conditions of each country in each year (GBD 2015 DALYs/HALE Collaborators 2016).

We report 95\% uncertainty intervals (UI) for each estimate, including rates, numbers of deaths, and DALYs. We estimated UIs by taking 1000 samples from the posterior distribution of each quantity and using the 25th and 975th-ordered draw of the uncertainty distribution.

\section{Results}

In 2015, 135,230 (95\% UI 123,034-148,184) individuals died from diabetes and 16,470 (95\% UI 13,977-18,961) from CKD-DM in the EMR. These numbers represent 216 and $179 \%$ increases in the number of deaths due to diabetes and CKD-DM, respectively, compared to 1990 . Figure 1 shows this increasing trend is not only for the number of deaths, but also for all ages and age-standardized mortality rates.

The total number of people with diabetes in the EMR in 2015 was 42.3 million (95\% UI 38.6-46.4 million). The highest prevalence rates of DM and CKD-DM were observed among those aged 70-79 years old; however, the highest numbers of cases were among the younger age groups. The patterns of prevalence were similar in both sexes (Fig. 2). 


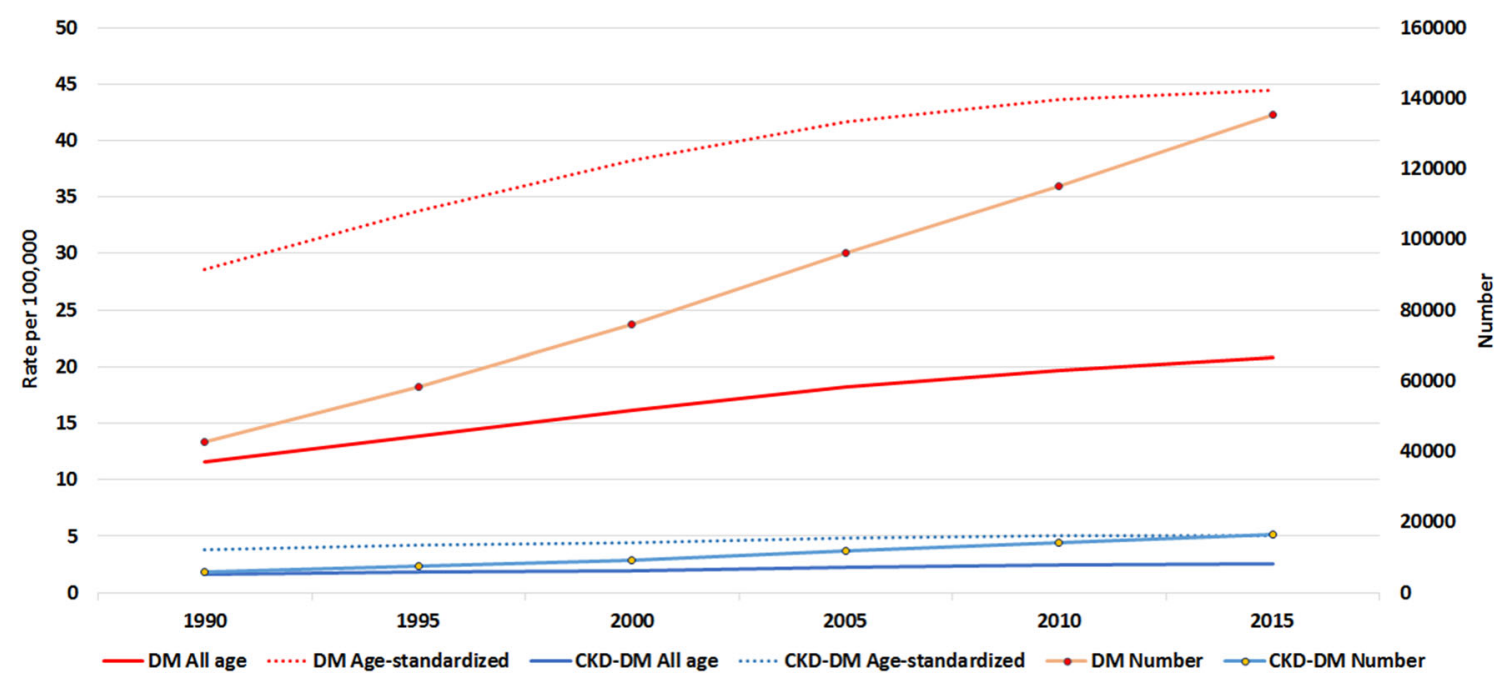

Fig. 1 Trend of number of deaths, all-age and age-standardized mortality of diabetes mellitus (DM) and chronic kidney disease due to diabetes mellitus (CKD-DM). (Global Burden of Disease 2015 study, Eastern Mediterranean Region, 1990-2015)

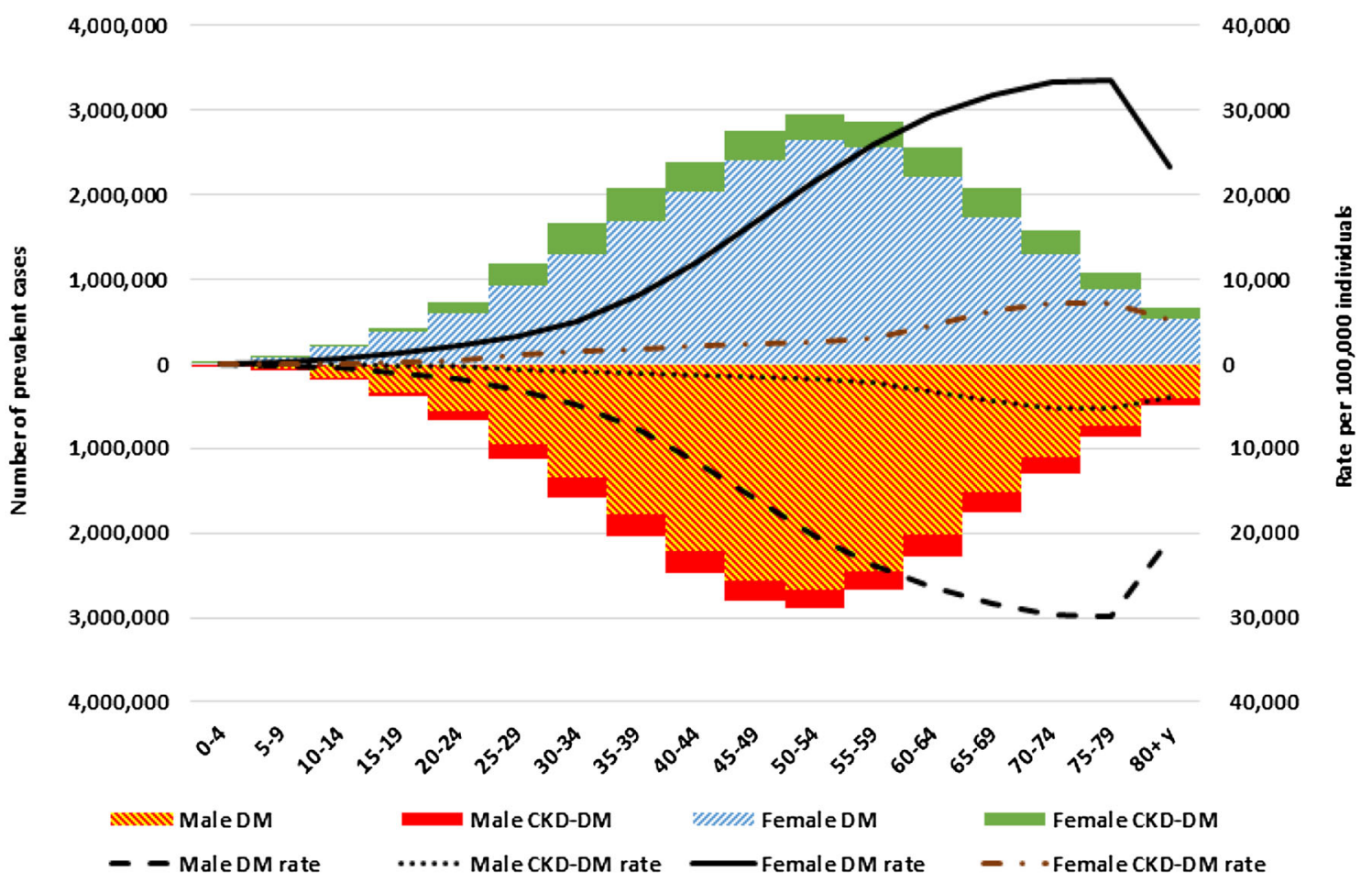

Fig. 2 Number and rate of prevalence cases of diabetes mellitus(DM) and chronic kidney disease due to diabetes mellitus(CKD-DM) in the Eastern Mediterranean Region, 2015. (Global Burden of Disease 2015 study, Eastern Mediterranean Region, 2015)

Total DALYs from diabetes were 6,708,539 (95\% UI $5,451,990-8,148,834)$ in 2015 and 2,285,117 (95\% UI $1,892,297-2,792,790$ ) in 1990. For CKD-DM, total DALYs were 568,351 (95\% UI 490,064-653,946) in 2015 and 234,194 (95\% UI: 201,911-272,837) in 1990. In 2015, the proportion of YLLs to DALYs was $45 \%$ for diabetes mellitus and $73 \%$ for CKD-DM.

The burden of diabetes mellitus as a percentage of total DALYs was $1.1 \%$ (95\% UI 1.0-1.3\%) in 1990 and increased to $2.9 \%$ (95\% CI $2.6-3.3 \%$ ) in 2015. These percentage were $0.11 \%$ (95\% UI $0.10-0.13 \%$ ) and $0.25 \%$ (95\% CI $0.22-0.28 \%$ ) for CKD-DM in 1990 and 2015, respectively. The age-standardized observed DALY rate of diabetes in the EMR was higher than in all other WHO regions. Also, observed DALY rates of diabetes in the EMR were higher than the expected (based on SDI) values (Fig. 3). However, observed DALY rates for CKD-DM were less than the expected rates (Fig. 4). 
Fig. 3 Rates of disabilityadjusted life years of diabetes mellitus per 100,000 population in the World Health Organization regions. AFR African region, EMR Eastern Mediterranean region, EUR European region, $A M R$ Region of Americas, SEAR Southeast Asia region, WPR Western Pacific region. (Global Burden of Disease 2015 study, World Health Organization regions, 2015)

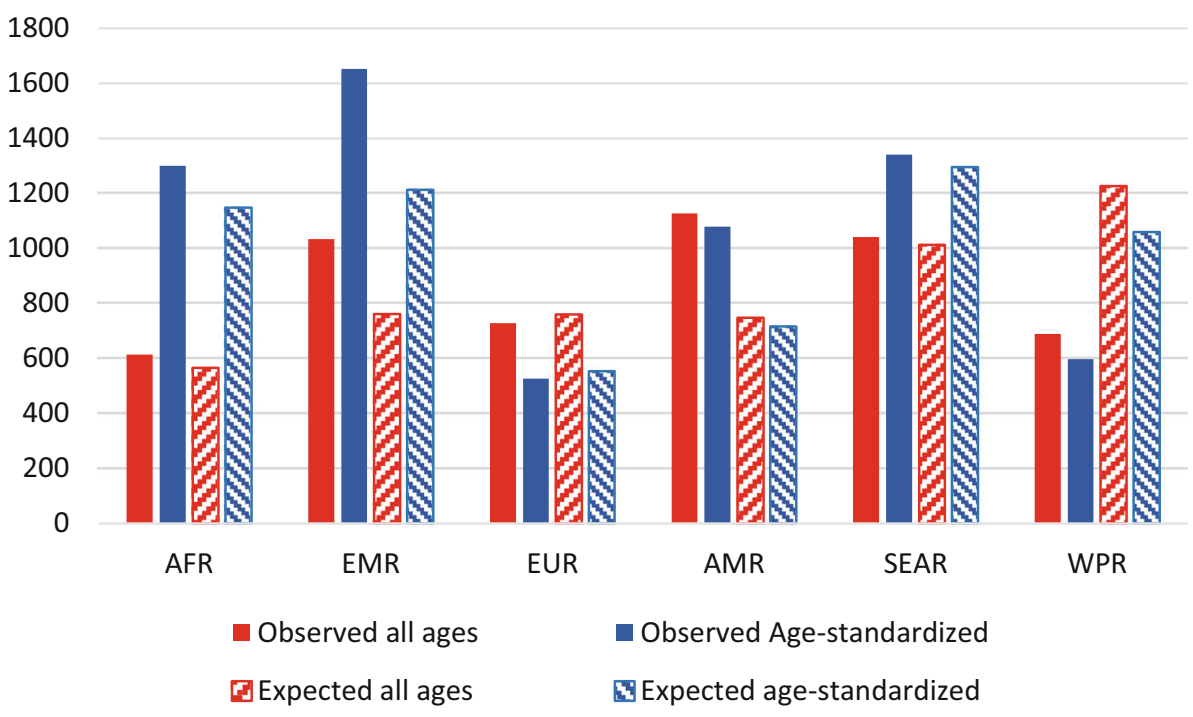

Fig. 4 Rates of disabilityadjusted life years of chronic kidney disease due to diabetes mellitus per 100,000 population in the World Health Organization regions. AFR African region, EMR Eastern Mediterranean region, EUR European region, $A M R$ Region of Americas, SEAR Southeast Asia region, WPR Western Pacific region. (Global Burden of Disease 2015 study, World Health Organization regions, 2015)

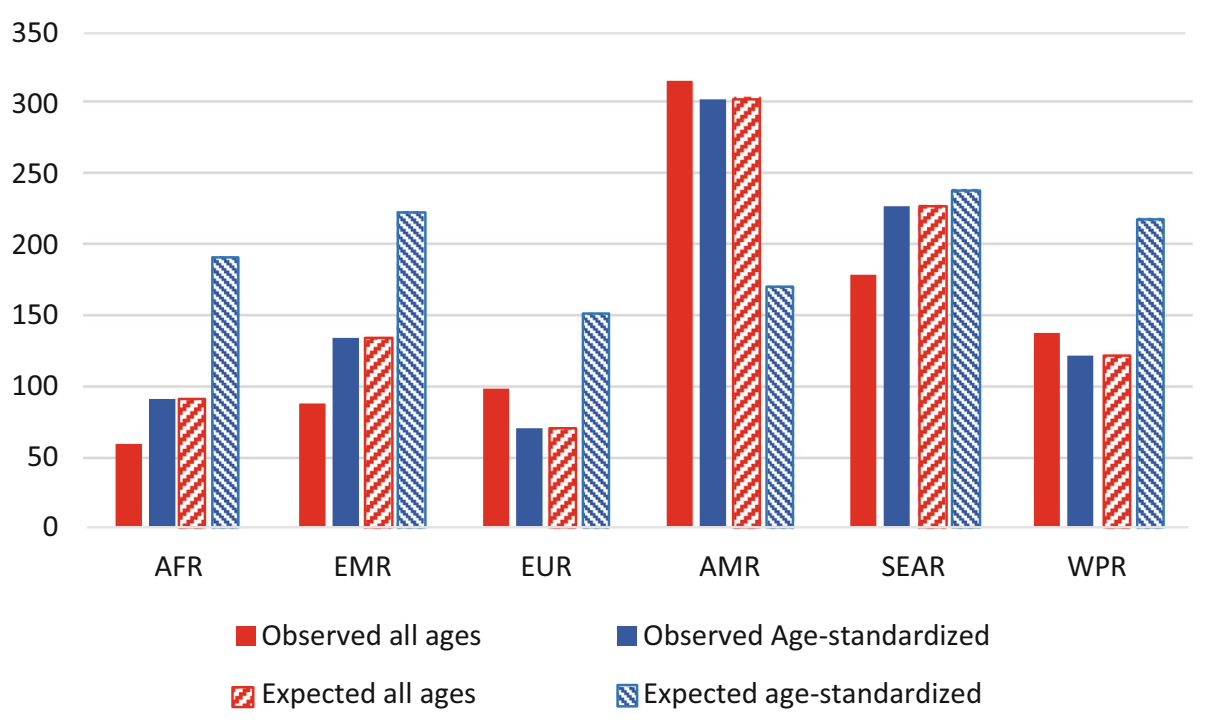

Table 1 lists DALY rates of diabetes and CKD-DM in each of the EMR countries in 2015. Morocco, Tunisia, and Bahrain had the highest DALY rates of diabetes, and Tunisia, Saudi Arabia, and Afghanistan had the highest DALY rates of CKD-DM.

\section{Discussion}

Our study showed that the burden of diabetes has increased considerably during the last 25 years in the EMR. This burden is higher than expected based on the demographic and social status of the countries in the region. Clearly, the region's health systems have not performed at the expected level, given their socio-demographic status, to control and prevent diabetes and CKD. This is in contrast to the European region and Western Pacific region, where observed levels are lower than expected levels. There are several potential reasons for such differenced: people in the EMR have lower perceived risk, and access to and quality of health care are lesser in this region (Mokdad et al. 2014; Moradi-Lakeh et al. 2016b) Our results call for urgent efforts to address the burden of diabetes in the region.

Several interventions have been suggested for prevention and control of diabetes. Although the effectiveness and cost-effectiveness of all interventions are not promising, there is evidence of several successful experiences around the world (Davies et al. 2017; Sun et al. 2017). For example, the National Diabetes Prevention Program showed successful changes in determinants of diabetes (Ely et al. 2017). Indeed, a multi-sectorial approach is needed to control and prevent diabetes in the region. WHO suggests the Package of Essential Non-communicable (PEN) Disease Interventions to be used in primary health care in low- 
Table 1 Disability-adjusted life years (DALYs) of diabetes mellitus and chronic kidney disease due to diabetes mellitus in the Eastern Mediterranean Region countries, 2015

\begin{tabular}{|c|c|c|c|c|c|c|c|c|c|c|c|c|}
\hline \multirow{4}{*}{$\begin{array}{l}\text { Location } \\
\text { Afghanistan }\end{array}$} & \multicolumn{6}{|c|}{ Chronic kidney disease due to diabetes mellitus } & \multicolumn{6}{|c|}{ Diabetes mellitus } \\
\hline & \multicolumn{3}{|l|}{ Male } & \multicolumn{3}{|c|}{ Female } & \multicolumn{3}{|l|}{ Male } & \multicolumn{3}{|c|}{ Female } \\
\hline & \multirow{2}{*}{$\frac{\text { Rate }}{137}$} & \multicolumn{2}{|c|}{$95 \% \mathrm{UI}$} & \multirow{2}{*}{$\frac{\text { Rate }}{204}$} & \multicolumn{2}{|c|}{$95 \%$ UI } & \multirow{2}{*}{$\frac{\text { Rate }}{735}$} & \multicolumn{2}{|c|}{$95 \% \mathrm{UI}$} & \multirow{2}{*}{$\frac{\text { Rate }}{1201}$} & \multicolumn{2}{|c|}{$95 \% \mathrm{UI}$} \\
\hline & & 73 & 222 & & 124 & 322 & & 550 & 970 & & 874 & 1645 \\
\hline Bahrain & 51 & 41 & 64 & 47 & 37 & 56 & 1569 & 1210 & 1988 & 1529 & 1188 & 1926 \\
\hline Djibouti & 111 & 61 & 215 & 82 & 52 & 146 & 1359 & 713 & 2691 & 856 & 483 & 1642 \\
\hline Egypt & 35 & 27 & 44 & 34 & 26 & 41 & 1265 & 1016 & 1570 & 1294 & 1029 & 1595 \\
\hline Iran & 75 & 56 & 97 & 66 & 51 & 83 & 908 & 685 & 1155 & 925 & 684 & 1191 \\
\hline Iraq & 25 & 19 & 32 & 28 & 21 & 35 & 1287 & 990 & 1613 & 1494 & 1157 & 1926 \\
\hline Jordan & 130 & 104 & 157 & 113 & 94 & 135 & 1052 & 810 & 1343 & 956 & 715 & 1242 \\
\hline Kuwait & 49 & 39 & 61 & 51 & 40 & 62 & 663 & 475 & 889 & 629 & 453 & 839 \\
\hline Lebanon & 70 & 47 & 101 & 86 & 60 & 117 & 1232 & 923 & 1585 & 1280 & 932 & 1627 \\
\hline Libya & 117 & 79 & 166 & 115 & 84 & 154 & 865 & 655 & 1112 & 1032 & 772 & 1334 \\
\hline Morocco & 122 & 85 & 174 & 124 & 87 & 168 & 1663 & 1265 & 2118 & 2061 & 1548 & 2611 \\
\hline Oman & 70 & 54 & 86 & 80 & 66 & 95 & 1203 & 916 & 1525 & 1168 & 888 & 1483 \\
\hline Pakistan & 114 & 86 & 141 & 67 & 54 & 81 & 895 & 699 & 1109 & 1091 & 847 & 1371 \\
\hline Palestine & 51 & 39 & 65 & 42 & 34 & 52 & 572 & 432 & 733 & 547 & 414 & 707 \\
\hline Qatar & 41 & 31 & 52 & 41 & 31 & 52 & 1015 & 748 & 1325 & 1077 & 801 & 1407 \\
\hline Saudi Arabia & 226 & 164 & 266 & 159 & 140 & 181 & 655 & 472 & 876 & 514 & 371 & 686 \\
\hline Somalia & 79 & 43 & 154 & 75 & 41 & 142 & 657 & 328 & 1303 & 527 & 265 & 1021 \\
\hline Sudan & 89 & 53 & 150 & 85 & 52 & 131 & 689 & 515 & 909 & 787 & 590 & 1013 \\
\hline Syria & 24 & 17 & 33 & 22 & 16 & 29 & 510 & 381 & 672 & 578 & 423 & 764 \\
\hline Tunisia & 264 & 196 & 354 & 183 & 137 & 238 & 1783 & 1396 & 2210 & 1527 & 1170 & 1920 \\
\hline UAE & 119 & 72 & 186 & 65 & 46 & 91 & 1231 & 890 & 1630 & 916 & 667 & 1196 \\
\hline Yemen & 86 & 50 & 143 & 107 & 62 & 184 & 536 & 386 & 719 & 792 & 571 & 1102 \\
\hline
\end{tabular}

Global Burden of Disease 2015 study, Eastern Mediterranean Countries, 2015

resource settings. PEN is a prioritized set of cost-effective interventions, tools, and aids to deliver an acceptable quality of care in the primary health care setting. Such interventions are feasible for adoption by most counties in the region (Zhang et al. 2016). For instance, Iran launched an adopted version, called IraPEN, with specific targets for prevention and control of non-communicable diseases. On the other hand, "Screen and Treat" strategies are unlikely to have a substantial impact to reduce the diabetes epidemic. Therefore, they should be complemented by population-wide approaches for effective diabetes prevention (Barry et al. 2017).

EMR countries are at different stages of prevention and control of diabetes; all high-income countries except Oman (Bahrain, Kuwait, Qatar, Saudi Arabia, and United Arab Emirates), some of the middle-income EMR countries (Iran, Jordan, Lebanon, and Tunisia), and none of the lowincome EMR countries have an operational policy, strategy, or plan of action for diabetes (WHO 2017).

CKD mortality increased in recent years in the region, underscoring the need for better treatment and management of blood pressure and diabetes. Diagnosis and control of diabetes and blood pressure are not optimal in the region. Early detection through screening of high-risk individuals is crucial to control blood pressure and diabetes and reduce diabetes and CKD burden and mortality. Although evidence is not strong enough to conclude that early diagnosis of diabetes will increase survival, treatment of impaired glucose tolerance or impaired fasting glucose, as well as lifestyle interventions, is associated with delayed progression to diabetes (Selph et al. 2015a, b). There is a need for more aggressive programs to control blood pressure and diabetes that include medical and preventive care approaches.

Access to and quality of medical care has a major impact on mortality from diabetes and CKD (Alegre-Diaz et al. 2016). Several studies have suggested that proper treatment might reduce complications and improve outcomes. Both diabetes and CKD require patients to adhere to long-term management of the condition (Brunton and Polonsky 2017). Unfortunately, not all the region's residents have equal access to quality medical care. It is 
possible that proper management of these conditions varies by county and has led to the observed increases in mortality.

Several studies have shown that obesity has rapidly increased in the region during the time period of this study (Mokdad et al. 2014, 2016). The studies have shown that inadequate physical activity and high body mass index are common in the region (El Bcheraoui et al. 2016). Moreover, dietary factors are among the major risk factors for diabetes and CKD (Moradi-Lakeh et al. 2016b; Yakoob et al. 2016). For example, low intake of whole grains, nuts and seeds, and fruit, and the consumption of processed food and red meats are known risk factor for diabetes; and high sodium intake is an important risk factor for CKD (Afshin et al. 2015; Moradi-Lakeh et al. 2016b). Diet has not improved much in the region during the study period (Afshin et al. 2015; Melaku et al. 2016; Otto et al. 2016). Moreover, there is only limited local information on dietary habits in the region (Afshin et al. 2015; Moradi-Lakeh et al. 2017). There is a need for programs to improve diet and physical activity and to control weight gain in the region to reduce the burden of diabetes as well as many other conditions. Only a few EMR countries have an operational policy, strategy, or plan of action to reduce obesity and physical inactivity (WHO 2017). The countries need to target different age groups, especially youth, to initiate sustainable changes in lifestyle. High intake of processed meat, sugar-sweetened beverages, and salt, and low intake of fruits and vegetables and whole grains need to be specifically addressed with regard to obesity, diabetes, and CKD-DM (Mokdad et al. 2016; Moradi-Lakeh et al. 2016a, 2017a; $\mathrm{Ng}$ et al. 2014).

Our study has a few limitations. For many countries with sparse data, estimates were driven by covariates in statistical modeling. The attributable effect of high body mass index (BMI) on ischemic heart disease, stroke, and diabetes was derived from prospective observational studies and meta-analyses. Our study does not account for variation within countries. We also do not have adequate data on access to and quality of health care in the region. More details on these limitations have been published elsewhere (Moradi-Lakeh et al. 2016b). On the other hand, we used new data for some countries, such as Saudi Arabia, which changed our estimates compared to GBD 2013 (El Bcheraoui et al. 2014; Moradi-Lakeh et al. 2016b).

\section{Conclusion}

Our study showed a large and increasing burden of diabetes in the region. This burden will increase with aging and growth of the population unless effective programs for control and prevention are put in place. Diabetes is a costly disease and most countries in the region spend a large percentage of their health resources on the disease. The region's financial and manpower resources are already stretched. Hence, there is an urgency to deal with diabetes and its consequences, and these efforts should be at the forefront of disease prevention and health promotion.

GBD 2015 Eastern Mediterranean Region Diabetes and Chronic Kidney Disease Collaborators Ali H. Mokdad, PhD (corresponding author), Institute for Health Metrics and Evaluation, University of Washington, Seattle, Washington, United States. Maziar MoradiLakeh, MD, Department of Community Medicine, Gastrointestinal and Liver Disease Research Center (GILDRC), Preventative Medicine and Public Health Research Center, Iran University of Medical Sciences, Tehran, Iran. Charbel El Bcheraoui, PhD, Institute for Health Metrics and Evaluation, University of Washington, Seattle, Washington, United States. Ibrahim Khalil, PhD, Institute for Health Metrics and Evaluation, University of Washington, Seattle, Washington, United States. Raghid Charara, MD, American University of Beirut, Beirut, Lebanon. Ashkan Afshin, MD, Institute for Health Metrics and Evaluation, University of Washington, Seattle, Washington, United States. Haidong Wang, PhD, Institute for Health Metrics and Evaluation, University of Washington, Seattle, Washington, United States. Michael Collison, BS, Institute for Health Metrics and Evaluation, University of Washington, Seattle, Washington, United States. Kristopher J. Krohn, BA, Institute for Health Metrics and Evaluation, University of Washington, Seattle, Washington, United States. Adrienne Chew, ND, Institute for Health Metrics and Evaluation, University of Washington, Seattle, Washington, United States. Farah Daoud, BA/BS, Institute for Health Metrics and Evaluation, University of Washington, Seattle, Washington, United States. Christopher D. Blosser, MD, Institute for Health Metrics and Evaluation, University of Washington, Seattle, Washington, United States. Leslie Cornaby, BS, Institute for Health Metrics and Evaluation, University of Washington, Seattle, Washington, United States. Kyle J. Foreman, PhD, Institute for Health Metrics and Evaluation, University of Washington, Seattle, Washington, United States; Imperial College London, London, UK. Nicholas J. Kassebaum, Institute for Health Metrics and Evaluation, University of Washington, Seattle, Washington, United States; Department of Anesthesiology \& Pain Medicine, Seattle Children's Hospital, Seattle, Washington, United States. Laura Kemmer, Institute for Health Metrics and Evaluation, University of Washington, Seattle, Washington, United States. Michael Kutz, BS, Institute for Health Metrics and Evaluation, University of Washington, Seattle, Washington, United States. Patrick Liu, BA, Institute for Health Metrics and Evaluation, University of Washington, Seattle, Washington, United States. Ben Zipkin, Institute for Health Metrics and Evaluation, University of Washington, Seattle, Washington, United States. Johan Ärnlöv, PhD, Department of Neurobiology, Care Sciences and Society, Division of Family Medicine and Primary Care, Karolinska Institutet, Stockholm, Sweden; School of Health and Social Studies, Dalarna University, Falun, Sweden. Kalkidan Hassen Abate, MS, Jimma University, Jimma, Oromia, Ethiopia. Alireza Ahmadi, PhD, Kermanshah University of Medical Sciences, Kermanshah, Iran. Hamid Ahmadieh, MD, Ophthalmic Research Center, Shahid Beheshti University of Medical Sciences, Tehran, Tehran, Iran; Department of Ophthalmology, Labbafinejad Medical Center, Tehran, Tehran, Iran. Muktar Beshir Ahmed, MPH, College of Health Sciences, Department of Epidemiology, ICT and e-Learning Coordinator, Jimma University, Jimma, Oromiya, Ethiopia. Ziyad Al-Aly, MD, Washington University in St. Louis, St. Louis, MO, United States. Khurshid Alam, PhD, Murdoch Childrens Research Institute, The University of Melbourne, Parkville, Victoria, Australia; The University of Melbourne, Melbourne, VIC, Austraila; The University 
of Sydney, Sydney, NSW, Austraila. Deena Alasfoor, MSc, Ministry of Health, Al Khuwair, Muscat, Oman. Raghib Ali, FRCP, University of Oxford, Oxford, UK. Reza Alizadeh-Navaei, PhD, Gastrointestinal Cancer Research Center, Mazandaran University of Medical Sciences, Sari, Mazandaran, Iran. Juma M. Alkaabi, FRCP, College of Medicine and Health Sciences UAEU, ALAIN UAE, Abu Zaby, UAE. Ala'a Alkerwi, PhD, Luxembourg Institute of Health (LIH), Strassen, Luxembourg. Rajaa Al-Raddadi, PhD, Joint Program of Family and Community Medicine, Jeddah, Makkah, Saudi Arabia. Khalid A. Altirkawi, MD, King Saud University, Riyadh, Saudi Arabia. Nelson Alvis-Guzman, PhD, Universidad de Cartagena, Cartagena de Indias, Colombia. Erfan Amini, MD, Uro-Oncology Research Center, Tehran University of Medical Sciences, Tehran, Iran; Non-communicable Diseases Research Center, Endocrinology and Metabolism Research Institute, Tehran University of Medical Sciences, Tehran, Iran. Nahla Anber, PhD, Mansoura University, Mansoura, Egypt. Palwasha Anwari, MD, Self-employed, Kabul, Kabul, Afghanistan. Solomon Weldegebreal Asgedom, PhD, Mekelle University, Mekelle, Tigray, Ethiopia. Tesfay Mehari Atey, MS, Mekelle University, Mekelle, Tigray, Ethiopia. Leticia Avila-Burgos, $\mathrm{PhD}$, National Institute of Public Health, Cuernavaca, Morelos, Mexico. Ashish Awasthi, PhD, Sanjay Gandhi Postgraduate Institute of Medical Sciences, Lucknow, Uttar Pradesh, India. Peter Azzopardi, MEpi, Department of Paediatrics, The University of Melbourne, Melbourne, VIC, Australia; Murdoch Childrens Research Institute, Melbourne, VIC, Australia; Wardliparingga Aboriginal Research Unit, South Australian Health and Medical Research Institute, Adelaide, SA; Centre for International Health, Burnet Institute, Melbourne, VIC, Australia. Till Bärnighausen, MD, Department of Global Health and Population, Harvard T H Chan School of Public Health, Harvard University, Boston, MA, United States; Africa Health Research Institute, Mtubatuba, KwaZulu-Natal, South Africa; Institute of Public Health, Heidelberg University, Heidelberg, Germany. Umar Bacha, PhD, School of Health Sciences, University of Management and Technology, Lahore, Punjab, Pakistan. Aleksandra Barac, PhD, Faculty of Medicine, University of Belgrade, Belgrade, Belgrade, Serbia. Shahrzad Bazargan-Hejazi, PhD, College of Medicine, Charles R. Drew University of Medicine and Science, Los Angeles, CA, United States; David Geffen School of Medicine, University of California at Los Angeles, Los Angeles, CA, United States. Neeraj Bedi, MD, College of Public Health and Tropical Medicine, Jazan, Saudi Arabia. Derbew Fikadu Berhe, MS, School of Pharmacy, Mekelle University, Mekelle, Tigray, Ethiopia; Groningen, Groningen, Netherlands. Addisu Shunu Beyene, MPH, College of Health and Medical Science, Haramaya University, Harar, Oromia, Ethiopia. Zulfiqar A. Bhutta, PhD, Centre of Excellence in Women and Child Health, Aga Khan University, Karachi, Pakistan; Centre for Global Child Health, The Hospital for Sick Children, Toronto, ON, Canada. Boris Bikbov, $\mathrm{PhD}$, Centre of Excellence in Women and Child Health, Haramaya University, Harar, Oromia, Ethiopia. Mulugeta M. Birhanu, MS, University of Groningen, UMCG, Groningen, Groningen, Netherlands; Mekelle University, Mekelle, Ayder 03, Ethiopia. Zahid A. Butt, PhD, Al Shifa Trust Eye Hospital, Rawalpindi Punjab, Pakistan. Lucero Cahuana-Hurtado, PhD, National Institute of Public Health, Cuernavaca, Morelos, Mexico. David O. Carpenter, MD, University at Albany, Rensselaer, New York, United States. Juan Jesus Carrero, PhD, Department of Medical Epidemiology and Biostatistics, Karolinska Institutet, Stockholm, Sweden. JeeYoung Jasmine/J Choi, PhD, Seoul National University Hospital, Seoul, South Korea; Seoul National University Medical Library, Seoul, South Korea. Hadi Danawi, PhD, Walden University, Minneapolis, Minnesota, United States. Samath D. Dharmaratne, MD, Department of Community Medicine, Faculty of Medicine, University of Peradeniya, Peradeniya, Sri Lanka. Eric L. Ding, ScD, Harvard $\mathrm{T} \mathrm{H}$ Chan School of Public Health, Harvard University, Boston, Massachusetts, United States. Shirin Djalalinia, PhD, Undersecretary for Research \& Technology, Ministry of Health \& Medical Education, Tehran, Tehran, Iran. Kerrie E. Doyle, PhD, RMIT University, Bundoora, VIC, Australia; Australian National University, Canberra, ACT, Australia. Hedyeh Ebrahimi, Non-Communicable Diseases Research Center, Tehran University of Medical Sciences, Tehran, Tehran, Iran; Liver and Pancreaticobiliary Diseases Research Center, Digestive Disease Research Institute, Shariati Hospital, Tehran University of Medical Sciences, Tehran, Tehran, Iran. Aman Yesuf Endries, MPH, Arba Minch University, Arba Minch, SNNPR, Ethiopia. Alireza Esteghamati, MD, Endocrinology and Metabolism Research Center, Tehran University of Medical Sciences, Tehran, Iran. Maryam S. Farvid, PhD Department of Nutrition, Harvard T.H. Chan School of Public Health, Harvard University, Boston, MA, United States; Harvard/MGH Center on Genomics, Vulnerable Populations, and Health Disparities, Mongan Institute for Health Policy, Massachusetts General Hospital, Boston, MA, United States. SeyedMohammad Fereshtehnejad, PhD, Department of Neurobiology, Care Sciences and Society (NVS), Karolinska Institutet, Stockholm, Sweden. Tesfaye Regassa Feyissa, MPH, Wollega University, Nekemte, Oromia, Ethiopia. Florian Fischer, PhD, School of Public Health, Bielefeld University, Bielefeld, North Rhine-Westphalia, Germany. Tsegaye Tewelde Gebrehiwot, MPH, Jimma University, Jimma, Oromia, Ethiopia. Philimon N. Gona, PhD, University of Massachusetts Boston, Boston, Massachusetts, United States. Sameer Vali Gopalani, MPH, Department of Health and Social Affairs, Government of the Federated States of Micronesia, Palikir, Pohnpei, Federated States of Micronesia. Bishal Gyawali, MPH, Aarhus University, Aarhus, Denmark; Kathmandu, Nepal. Nima Hafezi-Nejad, MD, Endocrinology and Metabolism Research Center, Tehran University of Medical Sciences, Tehran, Tehran, Iran. Randah Ribhi Hamadeh, DPhil, Arabian Gulf University, Manama, Bahrain. Samer Hamidi, DrPH, Hamdan Bin Mohammed Smart University, Dubai, UAE. Masako Horino, MPH, Bureau of Child, Family \& Community Wellness, Nevada Division of Public and Behavioral Health, Carson City, NV, United States. Mohamed Hsairi, MD, Department of Epidemiology, Salah Azaiz Institute, Tunis, Tunis, Tunisia. Mihajlo B. Jakovljevic, PhD, Faculty of Medical Sciences, University of Kragujevac Kragujevac, Central Serbia-Sumadija, Serbia, The Center for Health Trends and Forecasts, Institute for Health Metrics and Evaluation (IHME), The University of Washington, Seattle, Washington, United States. Aida Jimenez-Corona, $\mathrm{PhD}$, Department of Ocular Epidemiology and Visual Health, Institute of Ophthalmology Condede Valencia, Mexico City, Mexico, General Directorate of Epidemiology, Ministry of Health, Mexico City, Mexico. Denny John, MPH, International Center for Research on Women, New Delhi, Delhi, India. Jost B. Jonas, MD, Department of Ophthalmology, Medical Faculty Mannheim, Ruprecht-Karls-University Heidelberg, Mannheim, Germany, Germany. Amir Kasaeian, PhD, HematologyOncology and Stem Cell Transplantation Research Center, Tehran University of Medical Sciences, Tehran, Tehran, Iran; Endocrinology and Metabolism Population Sciences Institute, Tehran University of Medical Sciences, Tehran, Tehran, Iran. Andre Pascal Kengne, PhD, South African Medical Research Council, Cape Town, Western Cape, South Africa, University of Cape Town, Cape Town, Western Cape, South Africa. Ezra Belay Ketema, MS, Mekelle University, Mekelle, Ethiopia. Yousef Saleh Khader, ScD, Department of Community Medicine, Public Health and Family Medicine, Jordan University of Science and Technology, Irbid, Irbid, Jordan. Ejaz Ahmad Khan, MD, Health Services Academy, Islamabad, Punjab, Pakistan. Daniel Kim, DrPH, Department of Health Sciences, Northeastern University, Boston, Massachusetts, United States. Yun Jin Kim, PhD, Faculty of Chinese Medicine, Southern University College, Skudai, Johor, Malaysia. Yohannes Kinfu, PhD, Centre for Research and Action in Public Health, University of Canberra, Canberra, Australian Capital Territory, Australia. Katarzyna A. Kissimova-Skarbek, PhD, Jagiellonian University Medical College, Kraków, Poland. Ai Koyanagi, 
MD, Research and Development Unit, Parc Sanitari Sant Joan de Deu (CIBERSAM), Barcelona, Spain. Heidi J. Larson, PhD, Department of Infectious Disease Epidemiology, London School of Hygiene \& Tropical Medicine, London, UK; Institute for Health Metrics and Evaluation, University of Washington, Seattle, WA, United States. Anders Larsson, PhD, Department of Medical Sciences, Uppsala University, Uppsala, Sweden. Yongmei Li, PhD, San Francisco VA Medical Center, San Francisco, California, United States. Paulo A. Lotufo, DrPH, University of São Paulo, São Paulo, São Paulo, Brazil. Raimundas Lunevicius, PhD, Aintree University Hospital National Health Service Foundation Trust, Liverpool, UK; School of Medicine, University of Liverpool, Liverpool, UK. Azeem Majeed, MD, Department of Primary Care \& Public Health, Imperial College London, London, England, UK. Reza Malekzadeh, MD, Digestive Diseases Research Institute, Tehran University of Medical Sciences, Tehran, Iran; Digestive Diseases Research Institute, Tehran University of Medical Sciences, Tehran, Iran. Deborah Carvalho Malta, $\mathrm{PhD}$, Universidade Federalde Minas Gerais, Belo Horizonte, Minas Gerais, Brazil. Mohsen Mazidi, PhD, Key State Laboratory of Molecular Developmental Biology, Institute of Genetics and Developmental Biology, Chinese Academy of Sciences, Beijing, China. Ziad A. Memish, MD, Saudi Ministry of Health, Riyadh, Saudi Arabia, College of Medicine, Alfaisal University, Riyadh, Saudi Arabia. Walter, Mendoza, MD, United Nations Population Fund, Lima, Peru. Mubarek Abera Mengistie, MS, Jimma University, Jimma, Oromia, Ethiopia. George A. Mensah, MD, Center for Translation Research and Implementation Science, National Heart, Lung, and Blood Institute, National Institutes of Health, Bethesda, MD, United States. Haftay Berhane Mezgebe, MS, Mekelle University, Mekelle, Tigray, Ethiopia. Ted R. Miller, PhD, Pacific Institute for Research \& Evaluation, Calverton, MD, United States; Centre for Population Health, Curtin University, Perth, WA, Australia. Muktar Sano Kedir Mohammed, MS, Mizan Tepi University, Mizan Teferi, Ethiopia. Shafiu Mohammed, PhD, Health Systems and Policy Research Unit, Ahmadu Bello University, Zaria, Kaduna, Nigeria; Institute of Public Health, Heidelberg University, Heidelberg, Baden Wuettemberg, Germany. Ulrich O. Mueller, PhD, Federal Institute for Population Research, Wiesbaden, Germany. Gabriele Nagel, PhD, Ulm University, Ulm, Germany. Cuong Tat Nguyen, MSc, Institute for Global Health Innovations, Duy Tan University, Da Nang, Vietnam. Quyen Le Nguyen, MD, Institute for Global Health Innovations, Duy Tan University, Da Nang, Vietnam. Vuong Minh Nong, MSc, Institute for Global Health Innovations, Duy Tan University, Da Nang, Vietnam. Jean Jacques N. Noubiap, MD, University of Cape Town, Cape Town, Western Cape, South Africa, Medical Diagnostic Centre, Yaounde, Centre, Cameroon. Felix Akpojene Ogbo, MPH, Centre for Health Research, Western Sydney University, Sydney, New South Wales, Australia. Alberto Ortiz, PhD, IIS-Fundacion Jimenez Diaz-UAM, Madrid, Spain. Erika Ota, PhD, St. Luke's International University, Tokyo, Tokyo, Japan. Tejas Patel, MD, Mount Sinai Health System, New York, NY, United States. Jonathan Pearson-Stuttard, MD, Imperial College London, London, UK; Liverpool, UK. Norberto Perico, MD, IRCCS-Istituto di Ricerche Farmacologiche Mario Negri, Bergamo, Italy. Max Petzold, PhD, Health Metrics Unit, University of Gothenburg, Gothenburg, Sweden; University of the Witwatersrand, Johannesburg, South Africa. Farhad Pishgar, Non-Communicable Diseases Research Center, Tehran University of Medical Sciences, Tehran, Tehran, Iran; Uro-Oncology Research Center, Tehran University of Medical Sciences, Tehran, Tehran, Iran. Farshad Pourmalek, PhD, University of British Columbia, Vancouver, British Columbia, Canada. Mostafa Qorbani, $\mathrm{PhD}$, Non-Communicable Diseases Research Center, Alborz University of Medical Sciences, Karaj, Iran. Vafa Rahimi-Movaghar, MD, Sina Trauma and Surgery Research Center, Tehran University of Medical Sciences, Tehran, Tehran, Iran. Rajesh Kumar Rai, MPH, Society for Health and Demographic Surveillance, Suri, West Bengal,
India. Saleem M. Rana, PhD, Contech School of Public Health, Lahore, Punjab, Pakistan; Contech International Health Consultants, Lahore, Punjab, Pakistan. David Laith Rawaf, MD, MBBS, WHO Collaborating Centre, Imperial College London, London, UK, North Hampshire Hospitals, Basingstroke, UK, University College London Hospitals, London, UK. Salman Rawaf, MD, Imperial College London, London, UK. Giuseppe Remuzzi, MD, IRCCS-Istituto di Ricerche Farmacologiche Mario Negri, Bergamo, Italy; Azienda Socio-Sanitaria Territoriale, Papa GiovanniXXIII, Bergamo, Italy; Department of Biomedical and Clinical Sciences "L. Sacco", University of Milan, Milan, Italy. Andre M. N. N. Renzaho, PhD, Western Sydney University, Locked Bag 1797, Penrith 2751, NSW, Australia; NSW, Australia. Satar Rezaei, PhD, School of Public Health, Kermanshah University of Medical Sciences, Kermanshah, Iran. Gholamreza Roshandel, PhD, Golestan Research Center of Gastroenterology and Hepatology, Golestan University of Medical Sciences, Gorgan, Iran; Digestive Diseases Research Institute, Tehran University of Medical Sciences, Tehran, Iran. Dietrich Rothen bacher, MD, Institute of Epidemiology and Medical Biometry, Ulm University, Ulm, Germany. Mahdi Safdarian, MD, Sina Trauma \& Surgery Research Center, Tehran, Iran. Sare Safi, MS, Ophthalmic Epidemiology Research Center, Shahid Beheshti University of Medical Sciences, Tehran, Iran. Saeid Safiri, PhD, Managerial Epidemiology Research Center, Department of Public Health, School of Nursing and Midwifery, Maragheh University of Medical Sciences, Maragheh, Iran. Mohammad Ali Sahraian, MD, MS Research Center, Neuroscience Institute, Tehran University of Medical Sciences, Tehran, Iran. Payman Salamati, MD, Sina Trauma and Surgery Research Center, Tehran University of Medical Sciences, Tehran, Tehran, Iran. Abdallah M. Samy, PhD, Ain Shams University, Cairo, Egypt, Lawrence, Kansas, United States. Juan Ramon Sanabria, MD, J Edwards School of Medicine, Marshall Univeristy, Huntington, WV, United States; Case Western Reserve University, Cleveland, $\mathrm{OH}$, United States. Maria Dolores Sanchez-Niño, PhD, IIS-Fundacion Jimenez Diaz, Madrid, Madrid, Spain. Milena M. Santric Milicevic, $\mathrm{PhD}$, Institute of Social Medicine, Faculty of Medicine, University of Belgrade, Belgrade, Serbia, Serbia; Centre School of Public Health and Health Management, Faculty of Medicine, University of Belgrade, Belgrade, Serbia, Serbia. Benn Sartorius, PhD, Public Health Medicine, School of Nursing and Public Health, University of KwaZulu-Natal, Durban, South Africa; UKZN Gastrointestinal Cancer Research Centre, South African Medical Research Council (SAMRC), Durban, South Africa. Sadaf G. Sepanlou, PhD, Digestive Diseases Research Institute, Tehran University of Medical Sciences, Tehran, Tehran, Iran. Masood Ali Shaikh, MD, Independent Consultant, Karachi, Pakistan. Diego Augusto Santos Silva, PhD, Federal University of Santa Catarina, Florianopolis, SC-Santacatarina, Brazil. Dayane Gabriele Alves Silveira, MD, Brasília University, Brasília, DF, Brazil. Badr H. A. Sobaih, MD, King Saud University, Riyadh, Middleprovince, Saudi Arabia, Riyadh, Saudi Arabia. Rizwan Suliankatchi Abdulkader, MD, Ministry of Health, Kingdom of Saudi Arabia, Riyadh, Saudi Arabia. Rafael Tabarés-Seisdedos, PhD, Department of Medicine, University of Valencia, Incliva Health Research Institute and CIBERSAM, Valencia, Valencia, Spain. Arash Tehrani-Banihashemi, PhD, Preventive Medicine and Public Health Research Center, Iran University of Medical Sciences, Tehran, Tehran, Iran. Mohamad-Hani Temsah, MD, King Saud University, Riyadh, Saudi Arabia, King Faisal Specialist Hospital and Research Center, Riyadh, Saudi Arabia. Roman Topor-Madry, PhD, Institute of Public Health, Faculty of Health Sciences, Jagiellonian University Medical College, Kraków, Poland, Faculty of Health Sciences, Wroclaw Medical University, Wroclaw, Poland. Bach Xuan Tran, $\mathrm{PhD}$, Johns Hopkins University, Baltimore, Maryland, United States; Hanoi Medical University, Hanoi, Vietnam. Kingsley Nnanna Ukwaja, MD, Department of Internal Medicine, Federal Teaching Hospital, Abakaliki, Ebonyi State, Nigeria. Olalekan A. Uthman, 
$\mathrm{PhD}$, Warwick Medical School, University of Warwick, Coventry, UK. Job F. M. van Boven, PhD, University of Groningen, Groningen, Netherlands. Tolassa Wakayo, MS, Jimma University, Jimma, Oromia, Ethiopia. Andrea Werdecker, PhD, Competence Center Mortality-Follow-Up of the German National Cohort, Federal Institute for Population Research, Wiesbaden, Hessen, Germany. Abdulhalik Workicho, MPH, Jimma University, Jimma, Oromia, Ethiopia; Ghent University, Ghent, Belgium. Mohsen Yaghoubi, MA, University of Saskatchewan, Saskatoon, Saskatchewan, Canada; Tehran, Tehran, Iran. Yuichiro Yano, MD, Department of Preventive Medicine, Northwestern University, Chicago, Illinois, United States. Mehdi Yaseri, PhD, Tehran University of Medical Sciences, Tehran, Tehran, Iran; Ophthalmic Research Center, Shahid Beheshti University of Medical Sciences, Tehran, Tehran, Iran. Naohiro Yonemoto, MPH, Department of Biostatistics, School of Public Health, Kyoto University, Kyoto, Japan. Mustafa Z. Younis, DrPH, Jackson State University, Jackson, MS, United States. Anthony Lin Zhang, PhD, School of Health and Biomedical Sciences, RMIT University, Bundoora, VIC, Australia. Aisha O. Jumaan, PhD, Independent Consultant, Seattle, Washington, United States. Theo Vos, PhD, Institute for Health Metrics and Evaluation, University of Washington, Seattle, Washington, United States. Mohsen Naghavi, PhD, Institute for Health Metrics and Evaluation, University of Washington, Seattle, Washington, United States. Simon I. Hay, DSc, Oxford Big Data Institute, LiKa Shing Centre for Health Information and Discovery, University of Oxford, Oxford, UK; Institute for Health Metrics and Evaluation, University of Washington, Seattle, Washington, United States. Christopher J. L. Murray, DPhil, Institute for Health Metrics and Evaluation, University of Washington, Seattle, Washington, United States.

\section{Compliance with ethical standards}

Ethical statements The authors of this paper have complied with all ethical standards and do not have any conflicts of interest to disclose at the time of submission. The funding source played no role in the design of the study, the analysis and interpretation of data, and the writing of the paper.

Human participants and animals The study did not involve human participants and/or animals; therefore, no informed consent was needed.

Funding This research was funded by the Bill \& Melinda Gates Foundation.

Conflict of interest The authors declare that they have no conflicts of interest at this time.

Open Access This article is distributed under the terms of the Creative Commons Attribution 4.0 International License (http://crea tivecommons.org/licenses/by/4.0/), which permits unrestricted use, distribution, and reproduction in any medium, provided you give appropriate credit to the original author(s) and the source, provide a link to the Creative Commons license, and indicate if changes were made.

\section{References}

Afshin A, Micha R, Khatibzadeh S et al (2015) The impact of dietary habits and metabolic risk factors on cardiovascular and diabetes mortality in countries of the Middle East and North Africa in
2010: a comparative risk assessment analysis. BMJ Open 5(5):e006385. doi:10.1136/bmjopen-2014-006385

Alegre-Diaz J, Herrington W, Lopez-Cervantes M et al (2016) Diabetes and cause-specific mortality in Mexico City. N Engl J Med 375(20):1961-1971. doi:10.1056/NEJMoa1605368

Barry E, Roberts S, Oke J, Vijayaraghavan S, Normansell R, Greenhalgh T (2017) Efficacy and effectiveness of screen and treat policies in prevention of type 2 diabetes: systematic review and meta-analysis of screening tests and interventions. BMJ 356:i6538. doi:10.1136/bmj.i6538

Brunton SA, Polonsky WH (2017) Hot topics in primary care: medication adherence in type 2 diabetes mellitus: real-world strategies for addressing a common problem. J Fam Pract 66(4 Suppl):S46-S51

Davies MJ, Gray LJ, Troughton J et al (2017) A community-based primary prevention programme for type 2 diabetes mellitus integrating identification and lifestyle intervention for prevention: a cluster randomised controlled trial. Programme Grants for Applied Research, Southampton (UK)

Dieleman JL, Baral R, Birger M et al (2016) US spending on personal health care and public health, 1996-2013. JAMA 316(24):2627-2646. doi:10.1001/jama.2016.16885

Duncan BB, Inês Schmidt M, Cousin E et al (2017) The burden of diabetes and hyperglycemia in Brazil-past and present: findings from the Global Burden of Disease Study 2015. Diabetol Metab Syndr 9:18. doi:10.1186/s13098-017-0216-2

El Bcheraoui C, Basulaiman M, Tuffaha M et al (2014) Status of the diabetes epidemic in the Kingdom of Saudi Arabia, 2013. Int J Public Health 59(6):1011-1021. doi:10.1007/s00038-014-06124

El Bcheraoui C, Tuffaha M, Daoud F et al (2016) On your mark, get set, go: levels of physical activity in the Kingdom of Saudi Arabia, 2013. J Phys Act Health 13(2):231-238. doi:10.1123/ jpah.2014-0601

Ely EK, Gruss SM, Luman ET et al (2017) A national effort to prevent type 2 diabetes: participant-level evaluation of CDC's national diabetes prevention program. Diabetes Care. doi:10. 2337/dc16-2099

Foreman KJ, Lozano R, Lopez AD, Murray CJ (2012) Modeling causes of death: an integrated approach using CODEm. Popul Health Metr 10:1. doi:10.1186/1478-7954-10-1

GBD 2015 Causes of Death Collaborators (2016) Global, regional, and national life expectancy, all-cause mortality, and causespecific mortality for 249 causes of death, 1980-2015: a systematic analysis for the Global Burden of Disease Study 2015. Lancet 388(10053):1459-1544. doi:10.1016/S01406736(16)31012-1

GBD 2015 DALYs/HALE Collaborators (2016) Global, regional, and national disability-adjusted life-years (DALYs) for 315 diseases and injuries and healthy life expectancy (HALE), 1990-2015: a systematic analysis for the Global Burden of Disease Study 2015. Lancet 388(10053):1603-1658. doi:10.1016/S01406736(16)31460-X

GBD 2015 Disease and Injury Incidence and Prevalence Collaborators (2016) Global, regional, and national incidence, prevalence, and years lived with disability for 310 diseases and injuries, 1990-2015: a systematic analysis for the Global Burden of Disease Study 2015. Lancet 388(10053):1545-1602. doi:10. 1016/S0140-6736(16)31678-6

IDF (2015) IDF Diabetes Atlas. http://www.diabetesatlas.org/. Accessed 12 Jul 2017

Institute for Health Metrics and Evaluation (2016) GBD compare visualization tool. http://ihmeuw.org/421v. Accessed 30 April 2017

Melaku YA, Misganaw Temesgen A, Deribew A et al (2016) The impact of dietary risk factors on the burden of non- 
communicable diseases in Ethiopia: findings from the Global Burden of Disease study 2013. Int J Behav Nutr Phys Act 13(1):122. doi:10.1186/s12966-016-0447-x

Mokdad AH, Jaber S, Abdel Aziz MI et al (2014) The state of health in the Arab world, 1990-2010: an analysis of the burden of diseases, injuries, and risk factors. Lancet 383(9914):309-320. doi:10.1016/S0140-6736(13)62189-3

Mokdad AH, Forouzanfar MH, Daoud F et al (2016) Health in times of uncertainty in the eastern Mediterranean region, 1990-2013: a systematic analysis for the Global Burden of Disease Study 2013. Lancet Global Health 4(10):e704-e713. doi:10.1016/ S2214-109X(16)30168-1

Moradi-Lakeh M, El Bcheraoui C, Tuffaha M et al (2016a) The health of Saudi youths: current challenges and future opportunities. BMC Fam Pract 17:26. doi:10.1186/s12875-016-0425-z

Moradi-Lakeh M, Forouzanfar MH, El Bcheraoui C et al (2016b) High fasting plasma glucose, diabetes, and its risk factors in the eastern mediterranean region, 1990-2013: findings From the Global Burden of Disease Study 2013. Diabetes Care 40(1):22-29. doi:10.2337/dc16-1075

Moradi-Lakeh M, El Bcheraoui C, Afshin A, Daoud F et al (2017) Diet in Saudi Arabia: findings from a nationally representative survey. Public Health Nutr 20(6):1075-1081. doi:10.1017/ S1368980016003141

$\mathrm{Ng} \mathrm{M}$, Fleming T, Robinson M et al (2014) Global, regional, and national prevalence of overweight and obesity in children and adults during 1980-2013: a systematic analysis for the Global Burden of Disease Study 2013. Lancet 384(9945):766-781. doi:10.1016/S0140-6736(14)60460-8

Otto MC, Afshin A, Micha R et al (2016) The impact of dietary and metabolic risk factors on cardiovascular diseases and type 2 diabetes mortality in Brazil. PLoS One 11(3):e0151503. doi:10. 1371/journal.pone.0151503
Selph S, Dana T, Blazina I, Bougatsos C, Patel H, Chou R (2015a) Screening for type 2 diabetes mellitus: a systematic review for the U.S. Preventive Services Task Force. Ann Intern Med 162(11):765-776. doi:10.7326/M14-2221

Selph S, Dana T, Bougatsos C, Blazina I, Patel H, Chou R (2015b) Screening for abnormal glucose and type 2 diabetes mellitus: a systematic review to update the 2008 US Preventive Services Task Force Recommendation. U.S. Preventive Services Task Force Evidence Syntheses, formerly Systematic Evidence Reviews, Rockville (MD)

Sozmen K, Ünal B, Saidi O et al (2015) Cardiovascular risk factor trends in the Eastern Mediterranean region: evidence from four countries is alarming. Int J Public Health 60(Suppl 1):S3-S11. doi:10.1007/s00038-014-0610-6

Sun Y, You W, Almeida F, Estabrooks P, Davy B (2017) The effectiveness and cost of lifestyle interventions including nutrition education for diabetes prevention: a systematic review and meta-analysis. Journal of the Academy of Nutrition and Dietetics 117(3):404 e36-421 e36. doi:10.1016/j.jand.2016.11. 016

WHO (2017) Diabetes country profiles 2016. http://www.who.int/ diabetes/country-profiles/en/\#I. Accessed 10 June 2017

Yakoob MY, Micha R, Khatibzadeh S et al (2016) Impact of Dietary and Metabolic Risk Factors on Cardiovascular and Diabetes Mortality in South Asia: analysis From the 2010 Global Burden of Disease Study. Am J Public Health 106(12):2113-2125. doi:10.2105/AJPH.2016.303368

Zhang XH, Lisheng L, Campbell NR et al (2016) Implementation of World Health Organization Package of essential noncommunicable disease interventions (WHO PEN) for primary health care in low-resource settings: a policy statement from the world hypertension league. J Clin Hyperten 18(1):5-6. doi:10.1111/jch. 12749 